\title{
Optimal Settings of Residential Oil Burners
}

C. Ghiaus ${ }^{*}$, R. Belarbi, F. Allard

Laboratoire d'étude des phénomènes de transfert appliqués au bâtiment (LEPTAB), University of La Rochelle, France

*Corresponding author:

Cristian Ghiaus

LEPTAB, Univérsité de La Rochelle

Av. M. Crépeau

Tel: (33) 0546457248

Fax: (33) 0546458241

cristian.ghiaus@usa.net 


\title{
Optimal Settings of Residential Oil Burners
}

\begin{abstract}
Residential oil burners are capable of almost complete burning of the fuel oil, without visible smoke, when they are operated to deliver approximately $12 \% \mathrm{CO}_{2}$ in the flue gases. The positions of the air damper and of the combustion nozzle are adjusted at start-up and during operation in order to maximize the combustion efficiency. In practice, one factor at a time is varied, starting with the air damper. However, this method fails to detect the interaction between air excess and nozzle position and results in non-optimal settings. Optimal designed experiments allow obtaining local regression models and statistical analysis indicates if experiment augmentation is required. The air damper and combustion nozzle settings are changed in the direction of local gradient until a second order model, that contains the optimal point in its experimental region, is obtained. By using this approach, the gain in combustion efficiency may be 5\% higher than by using the classical approach.
\end{abstract}

\section{Introduction}

\subsection{Oil burner}

An oil burner combines oil and air under controlled conditions for combustion; the resulted energy is then delivered to the heating system of the building. Operated with excess air as low as $20 \%$, the oil burners are capable of almost complete burning of the fuel oil, without visible smoke. We try: 1) to maximize the burning efficiency and 2) to limit the pollutant concentration in the flue gases by controlling: 1) the smoke opacity, 2) the flue gases temperature and 3) the $\mathrm{CO}_{2}$ percentage. Small flue gases opacity is desired but it indicates that air excess may be too high; although low polluting, this operating condition is not energetically efficient. The temperature of the flue gases should be as low as possible but higher than the acid dew point $\left(160^{\circ} \mathrm{C}\right)$. The $\mathrm{CO}_{2}$ percentage in the flue gases is a function of air excess. The combustion efficiency depends on the combination of these three parameters.

Siegart [1] gives a simplified relation of the combustion efficiency:

$$
\eta=100-f \cdot\left(\frac{T_{f}-T_{a}}{C O_{2}}\right)
$$

where :

$\eta \quad$ combustion efficiency [\%];

$T_{f} \quad$ flue gases temperature [C];

$T_{a} \quad$ combustion air temperature [C];

$\mathrm{CO}_{2} \quad \mathrm{CO}_{2}$ concentration in flue gases [\%];

$f \quad$ coefficient depending on air excess $(f \cong 0.56$ for air excess of $20 \%)$.

The optimal settings of the oil burner, which consist in the positions of the air damper and of the combustion nozzle [Fig. 1, are when the combustion efficiency is maximal and the flue gases concentration in $\mathrm{CO}_{2}$ is less than $12 \%$. Preliminary adjustments of the air 
damper, based on the flame color and flue gases opacity, result in a near-optimal operating point. Then, the optimal settings are achieved by measuring the combustion efficiency and the concentration of $\mathrm{CO}_{2}$ in the flue gases.

The residential oil burner studied Fig. 1) presents an on-off combustion control system that starts and stops the fuel burner to satisfy the heat demand. The burner remains on until the controlled temperature of the outlet water reaches $85^{\circ} \mathrm{C}$; at this temperature, the burner shuts off. The burner again ignites at $75^{\circ} \mathrm{C}$. Although this combustion control system maintains the operating conditions in a limited domain, there is a systematic bias due to the variation of the outlet water temperature (Fig. 2a).

Our purpose is to elucidate the behavior of the burner near the optimal operating point by fitting an approximating function or model. The information needed is to be obtained by using as few experiments as possible.

\subsection{Operating point optimization}

Optimization consists in finding the set of operating conditions for the process variables that results in best performance. Generally, even rigorously formulated models have some unknown parameters, so experiments are used to acquire the data necessary for parameter estimation. In the case of oil burners, two settings are required: the positions of the air damper and of the combustion nozzle.

In practice, the one-factor-at-a-time procedure is used: first, the air damper position is changed and then the nozzle position. But the results obtained are misleading for two reasons: 1) the process parameters change in time with the increase of the outlet water temperature (Fig. 2a), and 2) the optimum point is not correctly localized due to the interaction between input variables. In Fig. $2 \mathrm{~b}$, for the nozzle position 0.8 , the maximum combustion efficiency found by modifying the air damper position is $86.6 \%$; then, by modifying the combustion nozzle position, the maximum efficiency found is $87.5 \%$. The settings are not optimal. Optimal designed experiments and response surfaces overcome these two problems.

Based on experiments, we may construct the response surfaces of combustion efficiency and $\mathrm{CO}_{2}$ concentration and use them to maximize the combustion efficiency. Response surface methodology is the most widely employed and successful optimization technique, developed in the early 1950s and initially applied in the chemical and process industries [2]. However, research on optimal experimental design, which allows us to obtain the measurements necessary to construct the response surfaces, was started in 1970s. The main idea of this approach is to design experiments intended to estimate the parameters and the adequacy of a given model [3]. Optimal experimental design makes optimization techniques applicable to real engineering systems.

\section{Response surface and the design of experiments}

The experiments for finding the optimal operating point are sequential and follow the formal description of steepest ascent and second order designs [4]. In the first stage, the experimenter decides on the input variables; in the case presented here, there are two: the positions of the air damper and of the nozzle. Then, based on experiments, local models are 
constructed. Following experiments are performed sequentially in the direction of steepest ascent. When the operating point is near the optimum, second order models are fitted; they may indicate a maximum in or near the experimental region.

In general, an experimental trial gives the measured values of responses, or output variables, $\mathbf{y}$, and of $m$ factors, or input variables, $\mathbf{u}$. In order to estimate a local model with $n$ parameters, at least $N=n$ trials are necessary at distinct points in the experimental domain. However, if the input-output relationship is blurred by errors, more trials will be required $(N>n)$ and regression will be used to fit the model; larger the degree of freedom, $v=N-n$, better the estimation. Statistical analysis can provide an estimation of both the variance of readings and of the residual sum of squares attributes to lack of fit.

\subsection{Experimental design domain and scaled variables}

Local models are obtained by varying the input variables, $\mathbf{u}$, in a given domain; the variation range is a trade off between model accuracy and experiment efficiency. It is convenient to scale and center the variables to lie between -1 and +1 . If the variation range is independent for each of the $m$ factors, the experimental domain, in terms of the scaled variables, is an $m$-dimensional cube; for $m=2$, the domain is a square. We consider hereafter that the input variables are scaled and centered.

\subsection{Multiple regression models}

For a system with $p$ inputs and one output, when we make $m$ experiments, we obtain $\mathbf{y}=\left[y_{1}, y_{2}, \ldots, y_{m}\right]$ values of the output of interest that correspond to $\mathbf{u}_{1}=\left[u_{1}, u_{2}, \ldots, u_{p}\right]_{1}$, $\mathbf{u}_{2}=\left[u_{1}, u_{2}, \ldots, u_{p}\right]_{2}, \ldots, \mathbf{u}_{m}=\left[u_{1}, u_{2}, \ldots, u_{p}\right]_{m}$ values of inputs. The general least square (or linear regression) problem is to find out the unknown parameters $\beta$ of the linear model [5]:

$$
y=\beta_{1} f_{1}(\mathbf{u})+\beta_{2} f_{2}(\mathbf{u})+\ldots+\beta_{n} f_{n}(\mathbf{u}),
$$

where $f_{1}, \ldots, f_{n}$ are known functions of $\mathbf{u}$. By transforming the input variables, we may note $\mathbf{x}_{1}=f_{1}(\mathbf{u}), \ldots, \mathbf{x}_{n}=f_{n}(\mathbf{u})$. Writing equation (1) for $m$ data pairs $\left\{\left(\mathbf{u}_{\mathrm{i}} ; y_{\mathrm{i}}\right), i=1, \ldots\right.$, $m$ \} by using matrix notation, we obtain:

$$
\mathbf{X} \beta=\mathbf{y},
$$

where

$$
\mathbf{X}=\left[\begin{array}{ccc}
f_{1}\left(\mathbf{u}_{1}\right) & \ldots & f_{n}\left(\mathbf{u}_{1}\right) \\
\ldots & \ldots & \ldots \\
f_{1}\left(\mathbf{u}_{m}\right) & \ldots & f_{n}\left(\mathbf{u}_{m}\right)
\end{array}\right], \beta=\left[\begin{array}{c}
\beta_{1} \\
\ldots \\
\beta_{n}
\end{array}\right] \text { et } \mathbf{y}=\left[\begin{array}{c}
y_{1} \\
\ldots \\
y_{m}
\end{array}\right] \text { are respectively the information }
$$

matrix of dimension $m \times n$, parameters (regression coefficients) vector of dimension $n \times 1$ and the output vector of dimension $m \times 1$. In order that equations (1) and (2) account for random noise and modeling error, an error vector $\mathbf{e}$ of dimensions $[m \times 1]$ is added:

$$
\mathbf{X} \beta+\mathbf{e}=\mathbf{y} .
$$


The least square problem is to find $b=\beta$ that minimizes the sum of squared error,

$$
\begin{aligned}
& E(\beta)=\sum_{i=1}^{m}\left(y_{i}-\sum_{j=1}^{n} \beta_{j} \cdot f_{j}\left(\mathbf{x}_{\mathbf{i}}\right)\right)^{2} \\
& =(\mathbf{y}-\mathbf{X} \beta)^{\mathbf{T}}(\mathbf{y}-\mathbf{X} \beta)=(\mathbf{y}-\hat{\mathbf{y}})^{\mathbf{T}}(\mathbf{y}-\hat{\mathbf{y}})=\mathbf{e}^{\mathbf{T}} \mathbf{e}
\end{aligned}
$$

where $\hat{\mathbf{y}}=\mathbf{X} b$ is the estimated output. If the model is linear in parameters and $\mathbf{X}^{T} \mathbf{X}$ is nonsingular, $b$ is unique and given by:

$$
b=\left(\mathbf{X}^{T} \mathbf{X}\right)^{-1} \mathbf{X}^{T} \mathbf{y} .
$$

\subsection{Confidence intervals on the parameter estimates}

Equation (5) gives the values of the regression coefficients (or estimated parameters), $b$. While the true value $\beta$ is fixed, the estimated value, $b$, varies randomly from estimation to estimation. In order to find the confidence intervals on $b$ we need the value of the error variance $\sigma^{2}$; we may find an estimation, $s^{2}$, of the variance, $\sigma^{2}$, by using the residual $\mathbf{e}=\mathbf{y}-\hat{\mathbf{y}}[6]:$

$$
s^{2}=\frac{1}{n-p} \mathbf{e}^{T} \mathbf{e}=\frac{1}{n-p} \sum_{i=1}^{n}\left(y_{i}-\hat{y}_{i}\right)^{2} .
$$

Then, the variance of the estimated parameter $b_{i}$ is:

$$
\operatorname{var} \beta_{i}=\frac{s}{\sqrt{\sum_{j=1}^{m} x_{i j}^{2}}}
$$

The $100(1-\alpha) \%$ confidence limits for $\beta_{i}$ may be estimated by using the $t$ distribution for $v=N-n$ degrees of freedom:

$$
\beta_{i}=b_{i} \pm t_{v, \alpha} \operatorname{var} \beta_{i}
$$

\subsection{D-optimal design of experiments}

Let us consider that we opted for a given model on a given domain. An optimal designed experiment minimizes the variance of parameter estimates for the chosen model on the given domain. Under the assumption of independent errors of constant variance $\sigma^{2}$, $E(\mathbf{e})=0$ and $E\left(\mathbf{e e}^{T}\right)=\sigma^{2} \mathbf{I}$ (Gauss-Markov conditions), the covariance matrix of the least squares estimates is: 


$$
\operatorname{var} b=\sigma^{2}\left(\mathbf{X}^{T} \mathbf{X}\right)^{-1}
$$

If interest is in the comparison of experimental designs, the value of $\sigma^{2}$ is not relevant since it is the same for all proposed designs for a specific experiment. The variance of $b_{j}$ is proportional to the $j$ th diagonal element of $\left(\mathbf{X}^{T} \mathbf{X}\right)^{-1}$. The determinant $\left|\left(\mathbf{X}^{T} \mathbf{X}\right)^{-1}\right|=1 /\left|\mathbf{X}^{T} \mathbf{X}\right|$ is called the generalized variance. Designs that maximize the value of the determinant of the Fischer's information matrix, $\mathbf{X}^{T} \mathbf{X}$, are called $D$-optimal ( $D$ for determinant). Maximizing the determinant $\left|\mathbf{X}^{T} \mathbf{X}\right|$ is equivalent to minimizing the determinant of the parameter covariance matrix [3].

The optimal design of experiments is based on the General Equivalence Theorem, which can be viewed as an application of the result that the derivatives are zero at an extremity of a function. The function (the local model chosen) depends on the measurements through the information matrix, $\mathbf{X}$. This theorem provides methods for asserting and checking the optimum location of the experimental points but it states nothing regarding their number. Practically, the number of experiments may be increased until the confidence multidimensional interval on the estimated parameters becomes acceptably small [3]. Hereafter, we give a method for testing if the augmentation of experiments is necessary.

The optimum design is found by searching over the design region. For simple problems, an analytical solution is sometimes possible [7]. In more complex situations, a sequential algorithm seeks the maximal increase of $\left|\mathbf{X}^{T} \mathbf{X}\right|$. The search over the continuous experimental domain is replaced by a search over a list of candidate points. This list, which is a coarse grid in the experimental region, frequently includes the points of the $D$ optimum continuous design. The problem is then to choose from the available grid the $m$ experimental points which maximize $\left|\mathbf{X}^{T} \mathbf{X}\right|$.

The product of the algorithm for optimum experiment design is a list of input combinations. The set of inputs must be sampled in such a way as to avoid systematic bias. Randomizing the sampling in the input multidimensional space results in avoiding the confounding of input effects with those due to omitted variables, which are nevertheless important.

The optimum design experiment is not self-evident (Fig. 3). The classical approach is to vary one factor at a time (while keeping the other unchanged). This design does not minimize the parameter estimation error and does not allow finding the interaction between variables. To illustrate these two aspects, let us consider two designs (Table 1 and Fig. 3 , for a linear model with interaction:

$$
y=b_{0}+b_{1} x_{1}+b_{2} x_{2}+b_{3} x_{1} x_{2} .
$$

Each measurement is affected by the same error, $\sigma$. Considering the theorem of variance applied in the case that all the variances are equal,

$$
\sigma_{\bar{x}}=\frac{\sigma_{x}}{\sqrt{n}},
$$


and solving the system of equations, we find for the classical design:

$b_{0}=\frac{1}{4}\left(v_{1}+v_{2}+v_{3}+v_{4}\right) \pm \frac{\sigma}{\sqrt{4}}, b_{1}=\frac{1}{4}\left(v_{2}-v_{1}\right) \pm \frac{\sigma}{\sqrt{2}}, b_{2}=\frac{1}{4}\left(v_{4}-v_{3}\right) \pm \frac{\sigma}{\sqrt{2}}$

and for the optimum design:

$$
\begin{aligned}
& b_{0}=\frac{1}{4}\left(w_{1}+w_{2}+w_{3}+w_{4}\right) \pm \frac{\sigma}{\sqrt{4}}, b_{1}=\frac{1}{4}\left(-w_{1}+w_{2}-w_{3}+w_{4}\right) \pm \frac{\sigma}{\sqrt{4}} \\
& b_{2}=\frac{1}{4}\left(-w_{1}-w_{2}+w_{3}-w_{4}\right) \pm \frac{\sigma}{\sqrt{4}}, b_{3}=\frac{1}{4}\left(w_{1}-w_{2}-w_{3}+w_{4}\right) \pm \frac{\sigma}{\sqrt{4}} .
\end{aligned}
$$

The classical design does not reveal the interaction term, $b_{3}$. The optimal design gives the necessary data to obtain the model and a smaller parameter estimation error. The determinant $\left|\mathbf{X}^{T} \mathbf{X}\right|$ based on the information matrix, $\mathbf{X}$, which is formed by the values of the variables of the model for every experiment (with rows corresponding to experiments and columns to variables in the model), gives us synthetically the same information (Fig. 3).

\subsection{The analysis of experiments and design augmentation}

The results of an experiment may be not sufficient for more reasons:

1. the model is inadequate;

2. the model does not give reproducible predictions.

In these cases, a solution is the augmentation of the number of experiments (Fig. 4). An adequate model should predict the results of new experiments and not change significantly its response surface when the number of experiments is increased (Fig. 5 and Fig. 6).

Inadequacies of the model are revealed during the course statistical analysis. The $F$ value, obtained by the analysis of variance, and the $R^{2}$ statistics give a measure of the model adequacy. If the model is inadequate, it means that the range of the experimental domain is too large. Excessive changes in the response suggest that the domain range should be reduced while failure to observe an effect suggests that a larger domain range would be necessary. Ideally, the ellipsoidal contour levels should be circles or circle sectors [Fig. 7].

\section{EXPERIMENTAL RESULTS AND DISCUSSION}

We applied response surface methodology to optimize the operating settings of the oil burner described in section 1 . Using a $D$-optimum design augmented from 8 to 13 trials (Fig. 4, we obtained the response surfaces for combustion efficiency and for $\mathrm{CO}_{2}$ concentration in flue gases. The positions of both the combustion nozzle and of the air damper may be continuously varied on a domain marked from 0 to 10 . A first experiment was achieved around the operating point of coordinates $\left[\begin{array}{ll}2.5 & 2.5\end{array}\right]$ in the experimental 
domain of [-1/2 1/2] and [-1 1$]$ for the positions of the air damper and of the combustion nozzle, respectively (Table 2 and Fig. 7 a).

When the response surface is evaluated considering the confidence intervals on the parameter estimates, two domain surfaces result, for the upper and lower limits of the confidence interval Fig. 5). A cross-section through these surfaces is depicted in Fig. 6. The facts that the contour levels do not change significantly and that the confidence interval does not decrease when the experiment is augmented indicate that eleven experiments would suffice.

The confidence interval indicates the precision to be used in the positioning of the optimum Fig. 8). We notice that the combustion efficiency may be estimated with a precision of $\pm 1 \%$ and the $\mathrm{CO}_{2}$ concentration with $\pm 0.5 \%$. These values should be considered when the contour lines of Fig. 7 are interpreted; two contour lines that differ with $1 \%$ in efficiency may not be distinct.

The gradient of the local quadratic model of the combustion efficiency, $\operatorname{grad}(\eta)=[-0.67-1.52]$, gives the direction for changing the operating point (Fig. 7a). The difference between the elements of the gradient vector indicates that the unitary variation for the second input variable should be reduced. A second trial is achieved in the point [3.0 2.75] by defining the input variables in the domain [-1/4 1/4] and [-1 1] for air damper and combustion nozzle, respectively. The resulted response surface is shown in Fig. $7 \mathrm{~b}$.

The model obtained based on the second experiment (Fig. 7b) indicates the optimum, which in the same time satisfies the imposed condition $\left(\mathrm{CO}_{2}\right.$ concentration less than $\left.12 \%\right)$. By changing the position of the combustion nozzle to 2.75 and of the air damper to 2.75 , the measured combustion efficiency becomes $90 \%$ and the $\mathrm{CO}_{2}$ concentration $11.7 \%$. This is the optimal operating point attainable by varying the input variables and represents a gain of $2.5 \%$ as compared with the classical approach that consists in varying one variable at a time Fig. 2p). Nevertheless, by changing the operating conditions (such as clearing up the boiler or using another type of fuel), the optimal point might change. In this case, the procedure for optimal settings should be applied again.

\section{CONCLUSION}

The experimental finding of optimal settings by using the response surfaces is very suitable to residential oil burners. The combustion efficiency depends on the positions of the nozzle and of the air damper upon a smooth function. This type of dependence makes the second order models appropriate; however, the slight curvature of the function, corroborated with the confidence intervals on the parameter estimates, introduces uncertainty in the exact optimum location. Practically, the optimal operating point may be situated anywhere in the region delimitated by the contour line corresponding to the maximum efficiency minus $1 \%$. For the same experimental effort, the gain in combustion efficiency may be 5\% higher than the value obtained by using the classical approach that consists in varying one input at a time. 


\section{REFERENCES}

[1] P. Chardot, P. Dupont Guide pratique du brûleur fioul, Sedit Edition, 1994 (ISBN 2236-00116-9)

[2] D.C. Montgomery, Introduction to Statistical Quality Control, John Wiley Sons, New York, 1996

[3] A.C. Atkinson, A.N. Donev, Optimum Experimental Designs, Oxford Science Publications, 1992

[4] G.E.P. Box, K.B. Wilson, On the experimental attainment of optimum conditions (with discussion), Journal of Royal Statistics Society, 13, 1-45, 1951

[5] J.-S. Jang, C.-T. Sun, E. Mizutani, Neuro-Fuzzy and Soft Computing, Pretince Hall, Upper Saddle River, 1997

[6] T.H. Wonnacott, R. J. Wonnacott, Statistique, Economica, Paris, 1995

[7] M.J. Box, N.R. Draper, Factorial designs, the $\left|F^{\prime} F\right|$ criterion and some related matters, Technometrics, 13, 731-742, 1971 


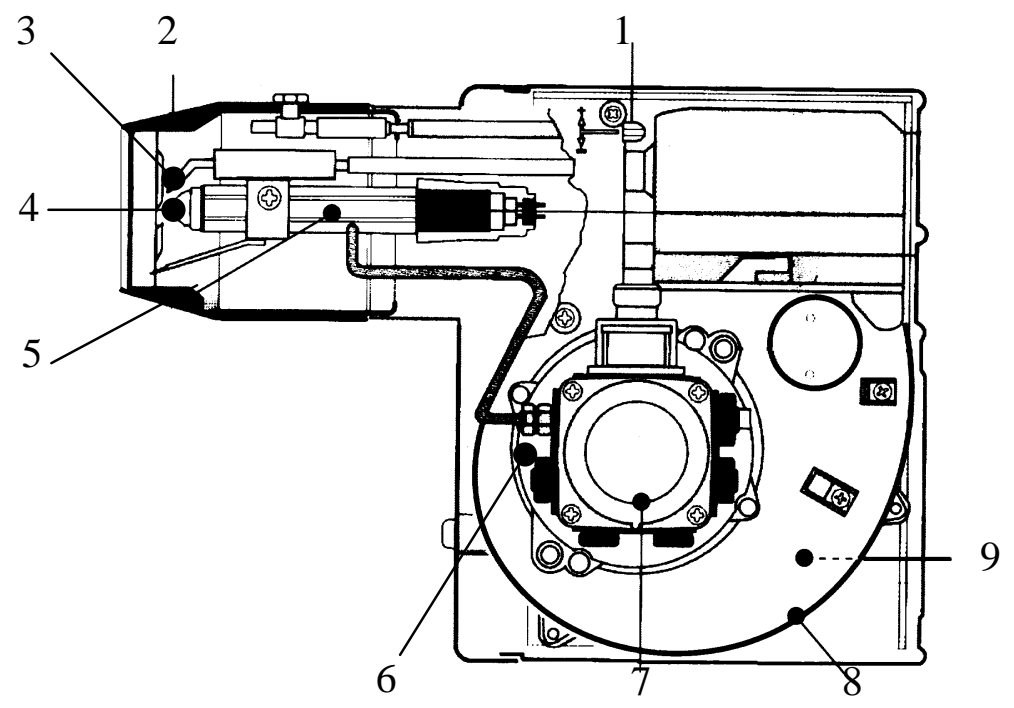

1. Combustion nozzle position 6. Motor

2. Retention head 7. Pump

3. Electrods 8. Fan

4. Combustion nozzle 9. Air damper

5. Fuel pipe

Fig. 1 Oil burner (adapted from [1]). 


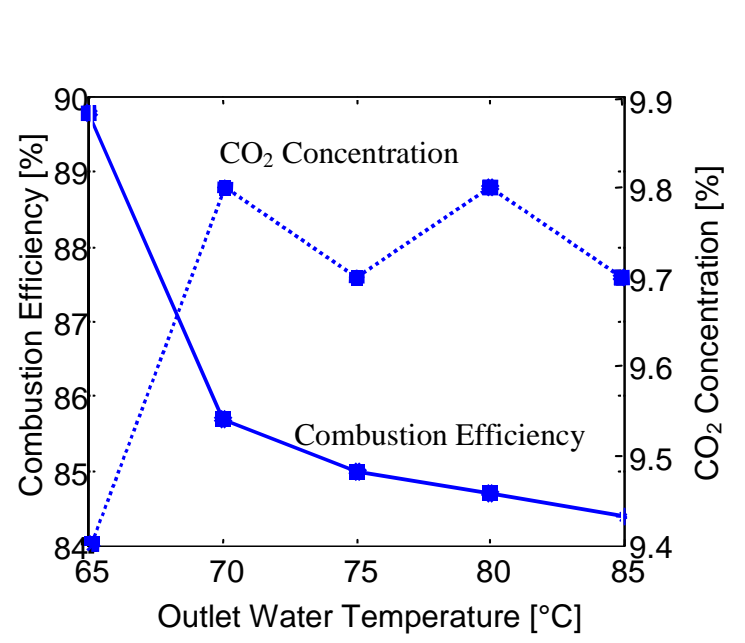

(a)

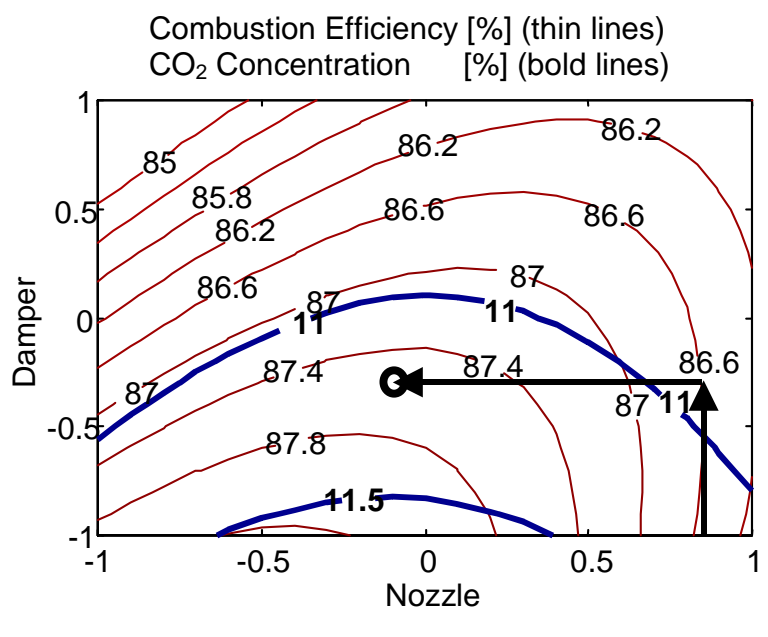

(b)

Fig. 2 One-factor-at-a-time method: a) Combustion efficiency changes with the outlet water temperature; b) Optimization. 


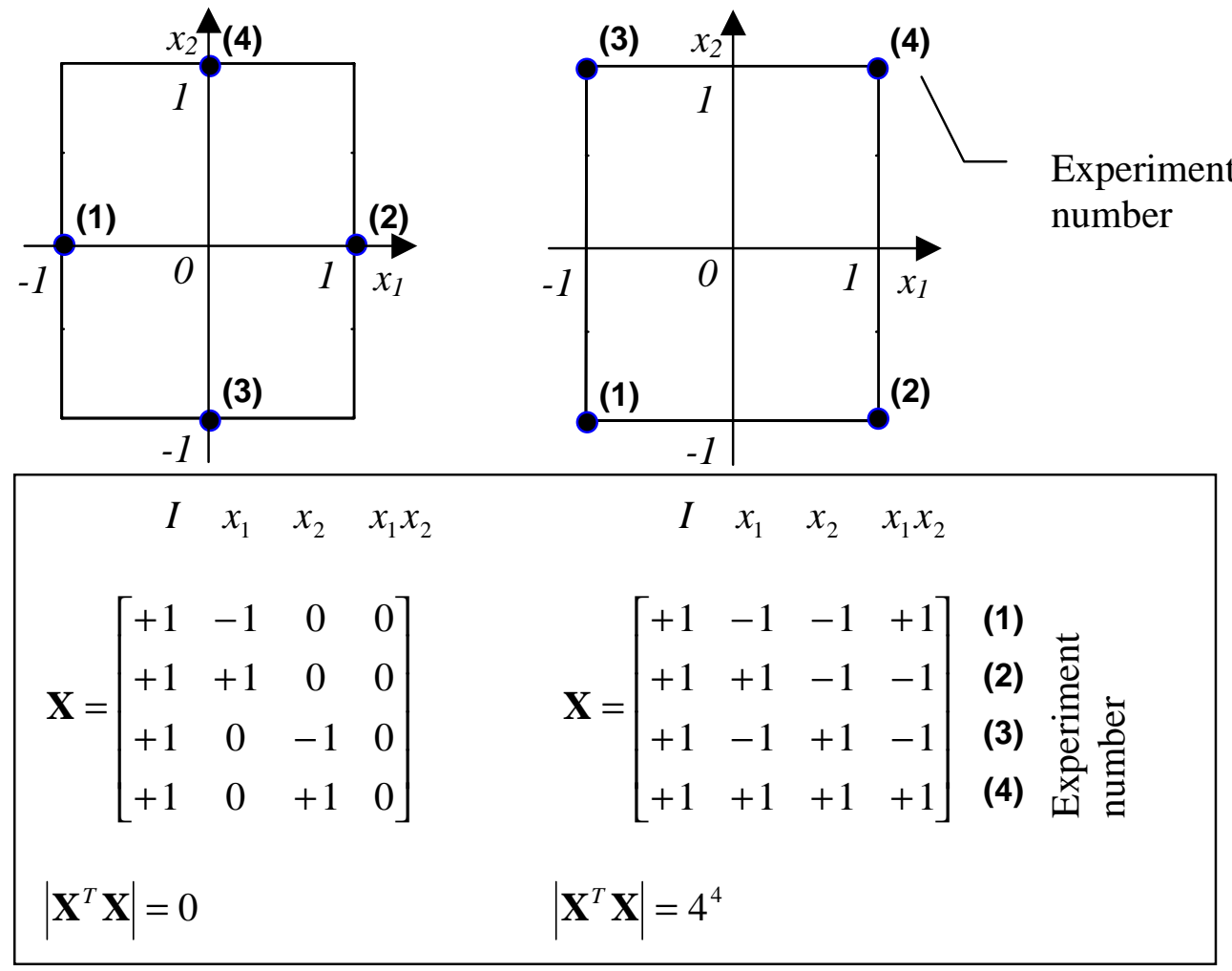

Fig. 3 Experiment design for a linear model of the form $y=b_{0}+b_{1} x_{1}+b_{2} x_{2}+b_{3} x_{1} x_{2}$. a) One-factor-at-a-time method. b) Optimum designed experiment. 

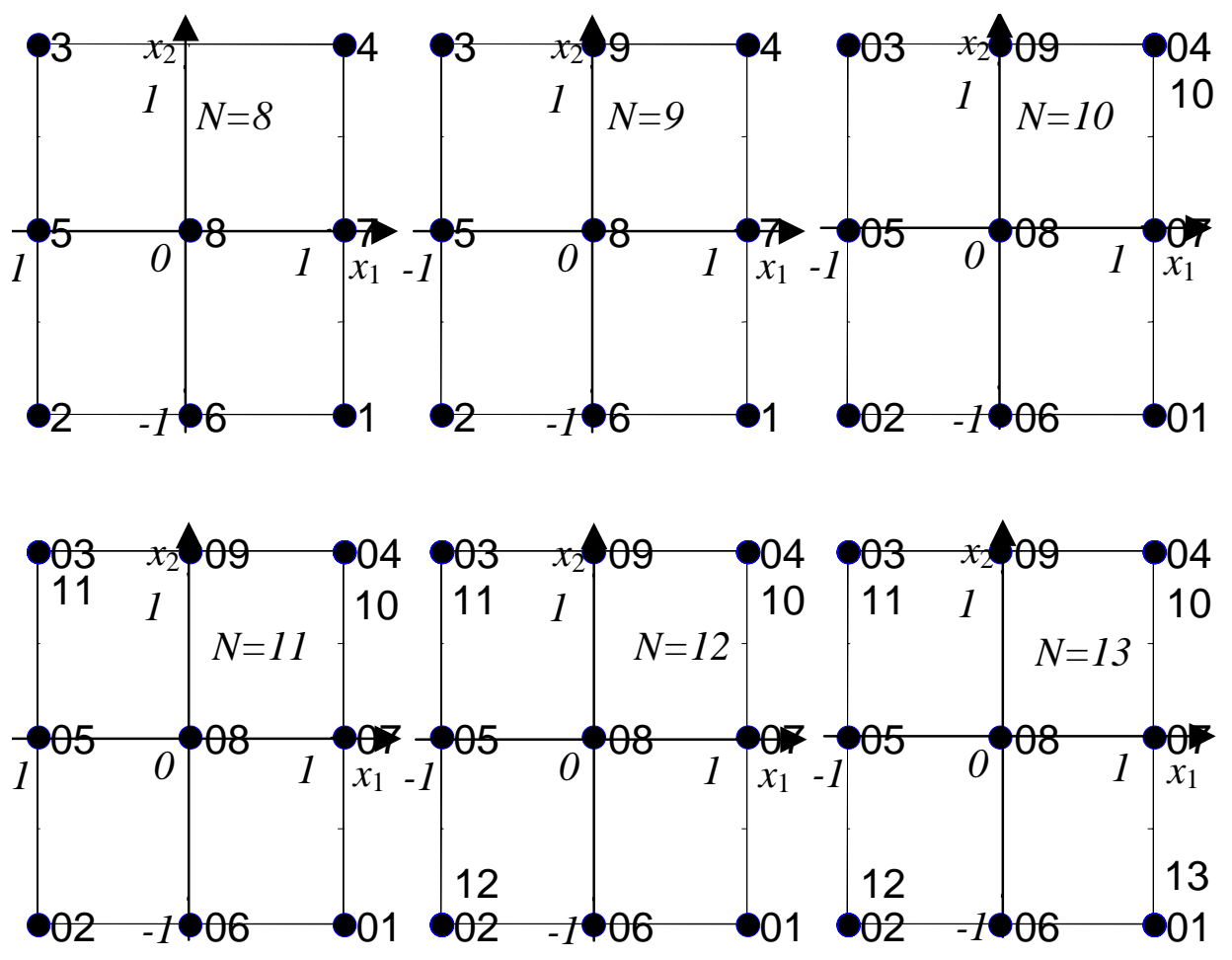

Fig. 4 Augmentation of $D$-optimal designed experiments for second order response surface from 8 to 13 trials. The trial number is indicated next to the points. 


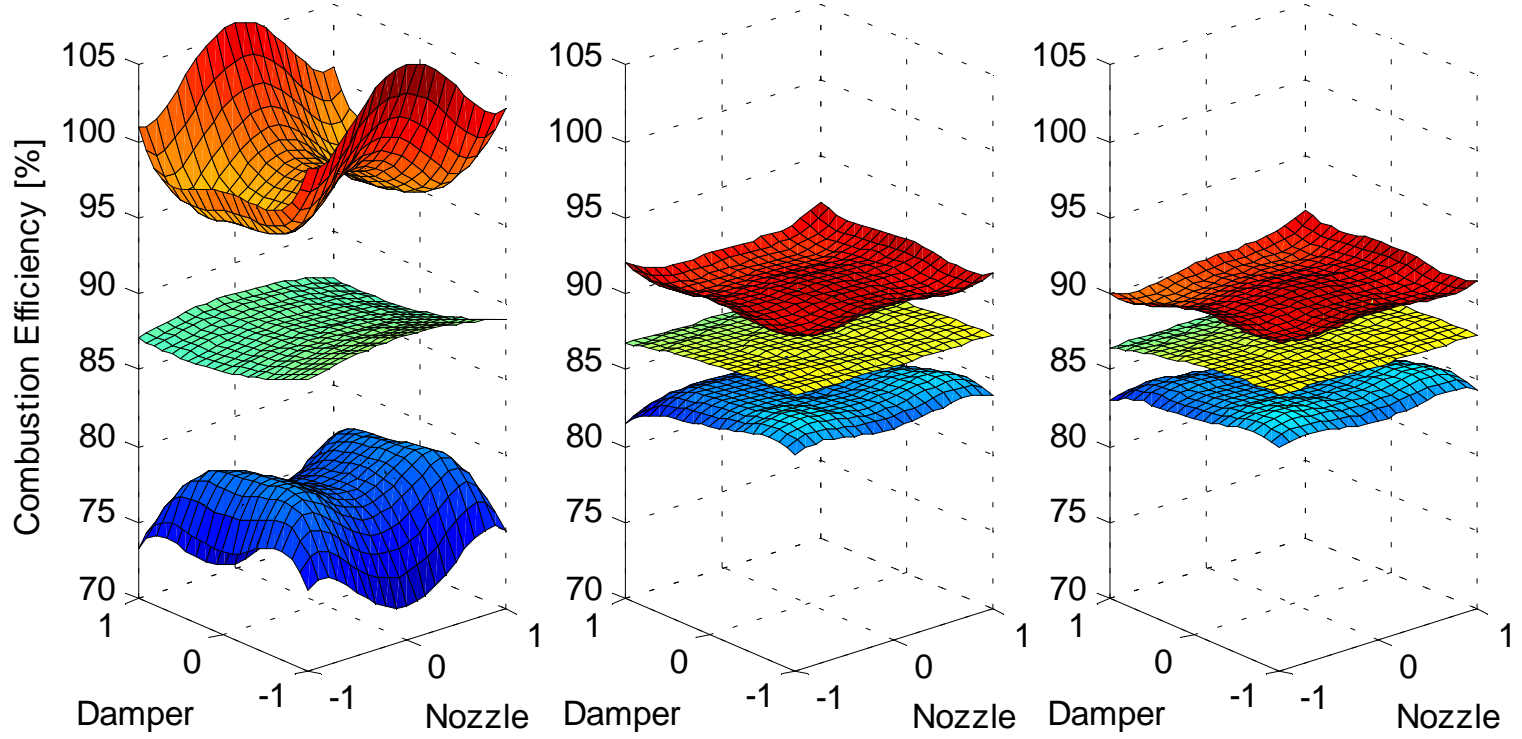

Fig. 5 Response surfaces and 95\% confidence interval for: a) 8 experimental points; 11 experimental points; $b) 12$ experimental points. 


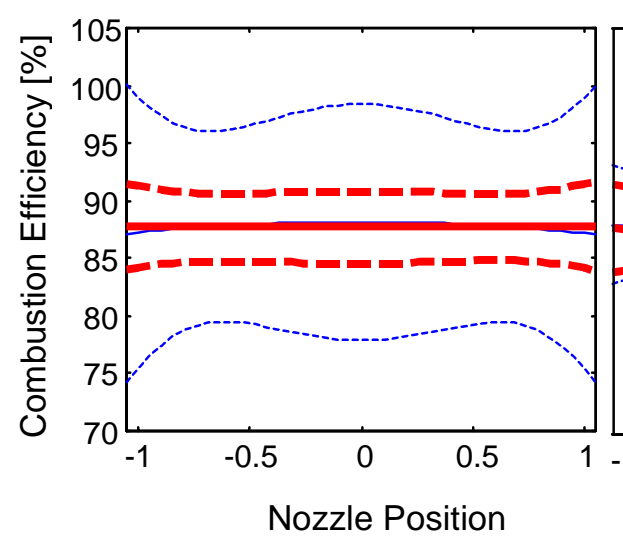

(a)

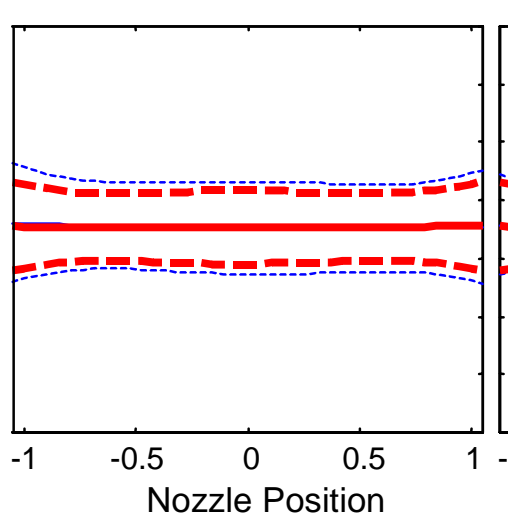

(b)

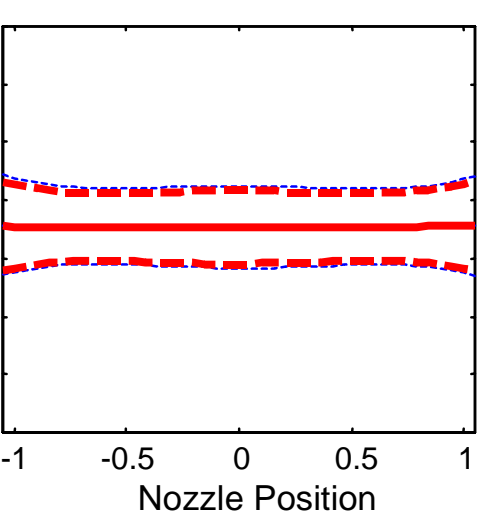

$(c)$

Fig. 6 Comparison of confidence intervals between models obtained by using 13 experiments (bold lines) and a) 8 experiments, b) 11 experiments and c) 12 experiments. The figures are cross-sections of the response surface for the damper position on zero. 


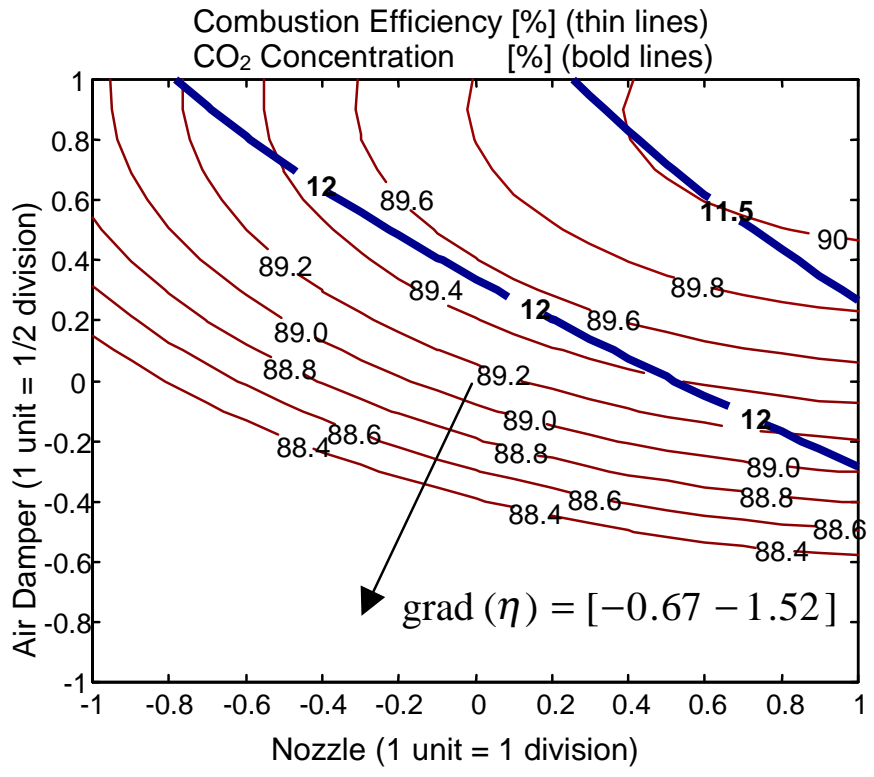

$(a)$

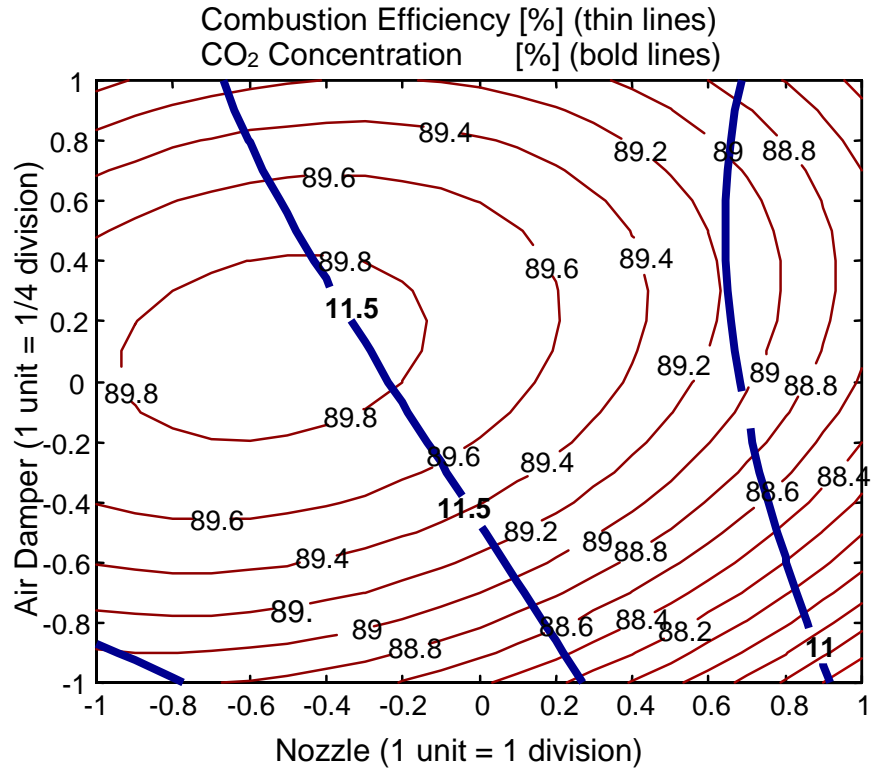

(b)

Fig. 7 Response surfaces for a) initial experiment and b) final experiment. The thin contour lines correspond to combustion efficiency and the bold contour lines to $\mathrm{CO}_{2}$ concentration. 

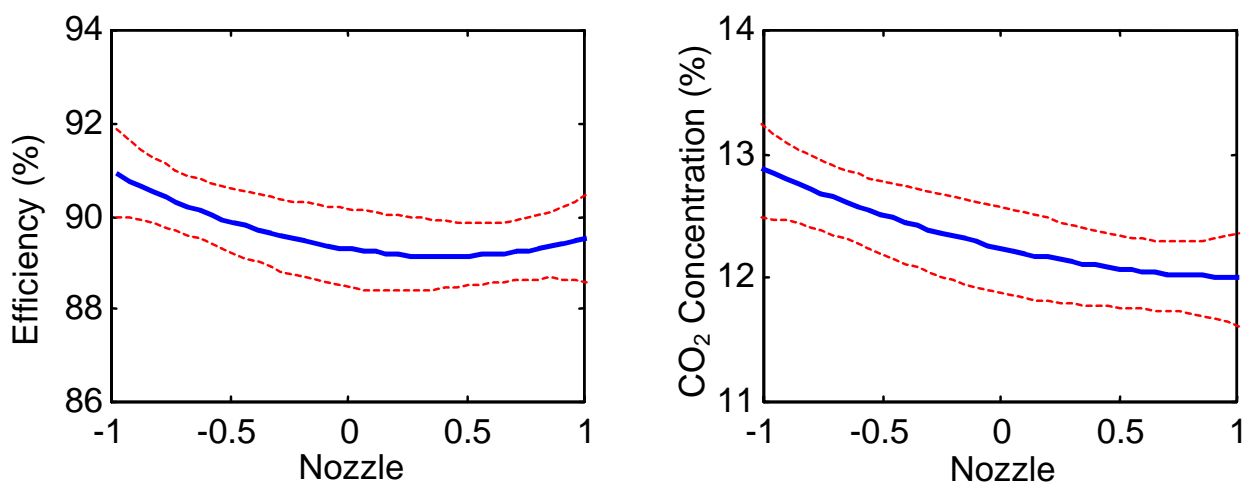

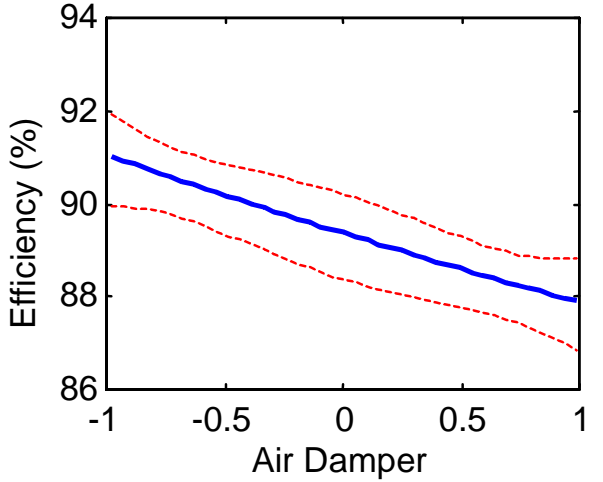

(a)

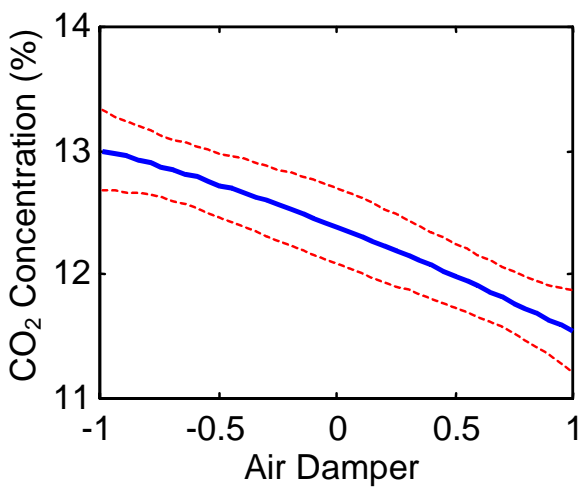

(b)

Fig. 8 Cross-section of the estimated models and their confidence intervals. a) combustion efficiency, b) $\mathrm{CO}_{2}$ concentration. 
Table 1 Two designs of experiments.

\begin{tabular}{|c|c|c|c|c|c|}
\hline \multicolumn{3}{|c|}{ Classical design } & \multicolumn{3}{|c|}{ Optimal design } \\
\hline$x_{1}$ & $x_{2}$ & $y$ & $x_{1}$ & $x_{2}$ & $y$ \\
\hline-1 & 0 & $v_{1} \pm \sigma$ & -1 & -1 & $w_{1} \pm \sigma$ \\
\hline+1 & 0 & $v_{2} \pm \sigma$ & +1 & -1 & $w_{2} \pm \sigma$ \\
\hline 0 & -1 & $v_{3} \pm \sigma$ & -1 & +1 & $w_{3} \pm \sigma$ \\
\hline 0 & +1 & $v_{4} \pm \sigma$ & +1 & +1 & $w_{4} \pm \sigma$ \\
\hline
\end{tabular}

Table 2 Experimental results obtained by varying the air damper and the combustion nozzle positions around the point $\left[\begin{array}{ll}2.5 & 2.5\end{array}\right]$ with $1 / 2$ and 1 division respectively.

\begin{tabular}{|c|c|c|c|c|c|}
\hline \multicolumn{4}{|c|}{ Inputs } & \multicolumn{2}{|c|}{ Outputs } \\
\hline \multicolumn{2}{|c|}{ Unscaled } & \multicolumn{2}{|c|}{ Scaled } & \multirow[b]{2}{*}{$\begin{array}{c}\text { Efficiency } \\
\%\end{array}$} & \multirow[b]{2}{*}{$\begin{array}{c}\mathrm{CO}_{2} \\
\% \\
\end{array}$} \\
\hline $\begin{array}{l}\text { Nozzle } \\
\text { div. }\end{array}$ & $\begin{array}{c}\text { Damper } \\
\text { div. }\end{array}$ & $\begin{array}{c}\text { Nozzle } \\
-\end{array}$ & $\begin{array}{c}\text { Damper } \\
-\end{array}$ & & \\
\hline 2.0 & 1.5 & -1 & -1 & 92.8 & 13.4 \\
\hline 2.0 & 2.5 & -1 & 0 & 90.5 & 12.8 \\
\hline 3.0 & 1.5 & 1 & -1 & 90.8 & 12.7 \\
\hline 3.0 & 3.5 & 1 & 1 & 88.3 & 11 \\
\hline 3.0 & 2.5 & 1 & 0 & 89.4 & 12.1 \\
\hline 2.5 & 1.5 & 0 & -1 & 90.7 & 13 \\
\hline 2.5 & 2.5 & 0 & 0 & 89.6 & 12.2 \\
\hline 2.0 & 3.5 & -1 & 1 & 89.2 & 12.1 \\
\hline 2.5 & 3.5 & 0 & 1 & 87.7 & 11.3 \\
\hline
\end{tabular}

